EPJ Web of Conferences 32, 01011 (2012)

DOI: 10.1051/epjconf/20123201011

(C) Owned by the authors, published by EDP Sciences, 2012

\title{
Benchmarking of electron cyclotron heating and current drive codes on ITER scenarios within the European Integrated Tokamak Modelling framework
}

\author{
L. Figini ${ }^{1, \text { a }}$, J. Decker ${ }^{2}$, D. Farina ${ }^{1}$, N. B. Marushchenko ${ }^{3}$, Y. Peysson ${ }^{2}$, E. Poli ${ }^{4}$, E. Westerhof ${ }^{5}$, and \\ ITM-TF contributors
}

1 Istituto di Fisica del Plasma CNR, EURATOM-ENEA-CNR Association, Milano, Italy

2 CEA, IRFM, F-13108, Saint-Paul-lez-Durance, France

3 Max Planck Institut für Plasmaphysik, EURATOM Association, Greifswald, Germany

4 Max Planck Institut für Plasmaphysik, EURATOM Association, Garching, Germany

5 FOM Institute DIFFER, Association EURATOM-FOM, Nieuwegein, Netherlands

\begin{abstract}
A number of electron cyclotron codes have been developed in different European laboratories, to describe the behavior of EC waves in realistic tokamak plasma scenarios, and to optimize EC heating and current drive efficiency in present, and future devices like ITER. Moreover, integrated modelling is gaining popularity, since it seems to be the optimum approach for modelling tasks as complex as the assessment of future large devices performances. The outcome is presented here of a benchmark exercise for a set of EC codes, performed within the framework of the European Integrated Tokamak Modelling Task Force, and based on a standard ITER scenario, considering multiple launching conditions of the EC waves. The advantages offered by integrated modelling are explored and exploited, and the sources of the small-but-still-present differences among the various codes results are investigated.
\end{abstract}

\section{Introduction}

Electron cyclotron (EC) resonance heating and current drive (H\&CD) will be one of the auxiliary systems available in ITER [1], and it is particularly important to have the EC system ready and optimized since the first phases of operation, in order to support scenarios development. To this goal, fast and accurate modelling tools are needed, and as an answer to this need, many laboratories developed EC codes, able to describe EC waves behavior in realistic tokamak plasma scenarios.

A few of these codes have participated in a past benchmarking study, but since that time more codes have become available, and some now include better physics models. In addition, integrated modelling has gained importance, being now the preferred approach for consistent and coherent results, when complex modelling tasks are needed, which requires the interaction of a large number of codes. Following this interest, a number of EC codes, have been recently integrated into the framework developed by the European Integrated Tokamak Modelling Task Force (EFDA ITM-TF). Within this framework, data containing physical information is exchanged between codes in a standardized way through generic data structures able to handle both simulated and experimental data.

Purpose of the work was to repeat the benchmarking exercise, now that several EC codes are available in the ITM framework, with the main objectives of both assessing the current state-of-the-art for the European ECH\&CD codes, and exploring the advantages provided by integrated modelling at the same time. To perform this study, a standard inductive H-mode scenario of ITER was chosen as

\footnotetext{
a e-mail: figini@ifp.cnr.it
} 


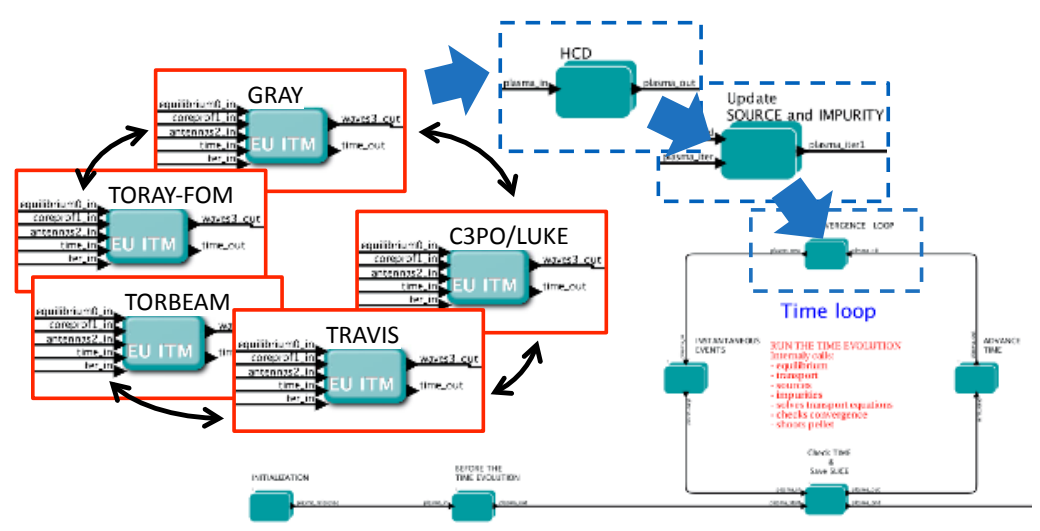

Fig. 1. Part of the European Transport Solver workflow, evidencing its multi-layered structure. All the codes involved in the benchmark share the same interface for data input/output, and can be interchanged in the workflow.

test case, and the launch of EC waves in different configurations, was considered both from the upper launcher (UL) and from the equatorial launcher (EL), according to the present design.

\section{The Integrated Tokamak Modelling framework}

The ITM-TF was born with the purpose of creating an environment where any complex simulation, requiring many different codes to interact, could be easily set up with a modular approach. Such a modular environment was developed assessing a common ontology for data structuring and workflow organization [2]. Within the ITM framework, the exchange of physics data happens through a defined data structure, with a standardized format. Interdependent physics information is grouped in complex elements, called "Consistent Physical Objects" (CPOs), which are the basic entities used for transferring both simulated and experimental data around a workflow. Moreover, a set of routines is made available in many programming languages to the code developers for handling the CPOs, reading and writing to the database. Codes modelling the same physics can be interchanged as modules of a same workflow, having the same input and output CPOs, i.e., all of them accept input data and provide the outputs with the same format. For building and managing the workflows, Kepler [3] has been adopted as the main tool. An example of the graphical representation of a data workflow in Kepler is shown in Figure 1. Each code is represented in the workflow by an actor, which takes care of retrieving the input data for the code from the database, and of collecting the results.

Providing a unique data structure for the exchange of physics information is not sufficient to ensure a correct and unambiguous communication among different codes: it is indeed equally important to have a clear and unique definitions of the reference systems adopted, of the mathematical definitions used to define all the quantities included in a CPO, and of the physical units used to measure them. A large effort was put in this benchmarking work, to check that all the codes involved were using the same conventions, and to fix and to clarify the definitions for the CPOs used.

\section{EC codes used}

The EC codes calculate the propagation of EC waves from the launching point (antenna) towards and within the plasma, the absorption of these waves by resonant electrons and the current driven in the plasma by this interaction. The codes can be classified on the basis of the models used for propagation, absorption, and current drive calculation. Differences may also exist in the way the transition from vacuum to plasma is handled. The main features of the five codes for ECH\&CD computation currently ported to the ITM framework, are listed in Table 1. Three of these codes, namely GRAY 
Table 1. Summary of the main features of the codes involved in the benchmark.

\begin{tabular}{|c|c|c|c|c|}
\hline Code name & Propagation model & Boundary model & Absorption model & Current drive model \\
\hline C3PO/LUKE & multi-ray, cold/ & Snell's law & \multicolumn{2}{|c|}{$\begin{array}{l}\text { bounce-averaged relativistic } \\
\text { Fokker-Planck, linear/quasi-linear }\end{array}$} \\
\hline GRAY & $\begin{array}{l}\text { warm dispersion } \\
\text { quasi-optical rays, } \\
\text { cold dispersion }\end{array}$ & polynomial SOL & $\begin{array}{l}\text { Fokker-Planck, linea } \\
\text { analytic, relativistic/ } \\
\text { weakly relativistic }\end{array}$ & $\begin{array}{l}\text { uasi-linear } \\
\text { adjoint, HS limit/ } \\
\text { momentum conservation }\end{array}$ \\
\hline TORAY-FOM & $\begin{array}{l}\text { multi-ray, cold/ } \\
\text { warm dispersion }\end{array}$ & unlimited SOL & analytic, relativistic & $\begin{array}{l}\text { adjoint, HS limit/ } \\
\text { momentum conservation }\end{array}$ \\
\hline TORBEAM & $\begin{array}{l}\text { Gaussian beam, } \\
\text { cold dispersion }\end{array}$ & Snell's law & $\begin{array}{l}\text { analytic, relativistic/ } \\
\text { weakly relativistic }\end{array}$ & $\begin{array}{l}\text { adjoint, HS limit/ } \\
\text { momentum conservation }\end{array}$ \\
\hline TRAVIS & $\begin{array}{l}\text { multi-ray, cold/ } \\
\text { warm dispersion }\end{array}$ & Snell's law & analytic, relativistic & $\begin{array}{l}\text { adjoint, momentum } \\
\text { conservation }\end{array}$ \\
\hline
\end{tabular}

[4], TORAY-FOM [5], and TORBEAM [6] had already been benchmarked in 2008 against other EC codes [7]. Since that benchmark, all the codes have been subject to changes to improve the description of various physics' aspects, e.g. the current drive model. Together with these three, two more codes have been recently ported to the ITM-TF framework, i.e. the TRAVIS code [8], and the C3PO/LUKE code package [9]. Absorption calculation in LUKE can be done using the standard linear theory or a quasi-linear model, with a 3D linearized bounce-averaged relativistic Fokker-Planck solver in the curvilinear coordinate system $(\psi, \theta, \phi)$, where $\psi$ is the poloidal magnetic flux, and $\theta$ and $\phi$ the poloidal and toroidal angles. The same coordinate system is used for ray-tracing in C3PO too. The other codes instead use a reference system in cartesian $(x, y, z)$ or cylindrical $(R, \phi, z)$ centered on the tokamak axis, and compute the absorption coefficient with an analytic relativistic model for Maxwellian distribution functions, while current drive is calculated solving the adjoint equation accounting for momentum conservation [10] (not yet included in the ITM version of TORAY-FOM), or a high speed limit model [11]. Geometric optics is used to calculate the propagation of independent rays in the codes C3PO, TORAY-FOM and TRAVIS, while TORBEAM uses a paraxial expansion of the complex eikonal to preserve diffraction and interference effects. The quasi-optical GRAY code traces rays to describe a Gaussian beam, in the framework of the complex eikonal approach. This latest approach prevents the use of Snell's law for describing the transition between vacuum and plasma propagation, and a smooth extrapolation of the density profile is applied.

In the ITM framework, all the five codes considered in this work share the same set of input and output CPOs: this allows to run them in the same workflow, with no need to perform pre- and postprocessing of the data separately for every code, avoiding a possible source of discrepancies.

\section{ITER as benchmarking case}

The plasma parameters and geometry used for this benchmarking study are those of a standard ITER scenario, known as "Scenario 2", used also for the benchmark in [7]. It is an H-mode scenario, with nominal vacuum toroidal field $B_{0}=5.3 \mathrm{~T}$ at major radius $R_{0}=6.2 \mathrm{~m}$, plasma current $I_{p}=15 \mathrm{MA}$, central electron density $n_{e, 0}=1.02 \times 10^{20} \mathrm{~m}^{-3}$, central electron temperature $T_{e, 0}=25 \mathrm{keV}$, and an effective ionic charge $Z_{\text {eff }} \sim 1.7$ roughly uniform throughout the plasma. The flux surfaces with rational safety factor $q=3 / 2$ and $q=2$, where the most harmful MHD modes are expected to arise, are located at a minor radius $\rho=0.81$ and $\rho=0.90$ respectively. Here $\rho$ is the square root of the normalized poloidal flux. Plasma geometry and kinetic profiles are shown in Figure 2.

In order to benchmark the codes in various conditions, three different launching conditions have been selected, each one with its own peculiarities, both from the equatorial and from the upper launcher. (i) Launch of a divergent gaussian beam from a mirror in the EL. In this case the absorption is expected to occur quite close to the plasma core. (ii) Same as the previous case, but with a larger toroidal angle, giving larger Doppler broadening, and allowing interaction at larger radii. (iii) Launch 

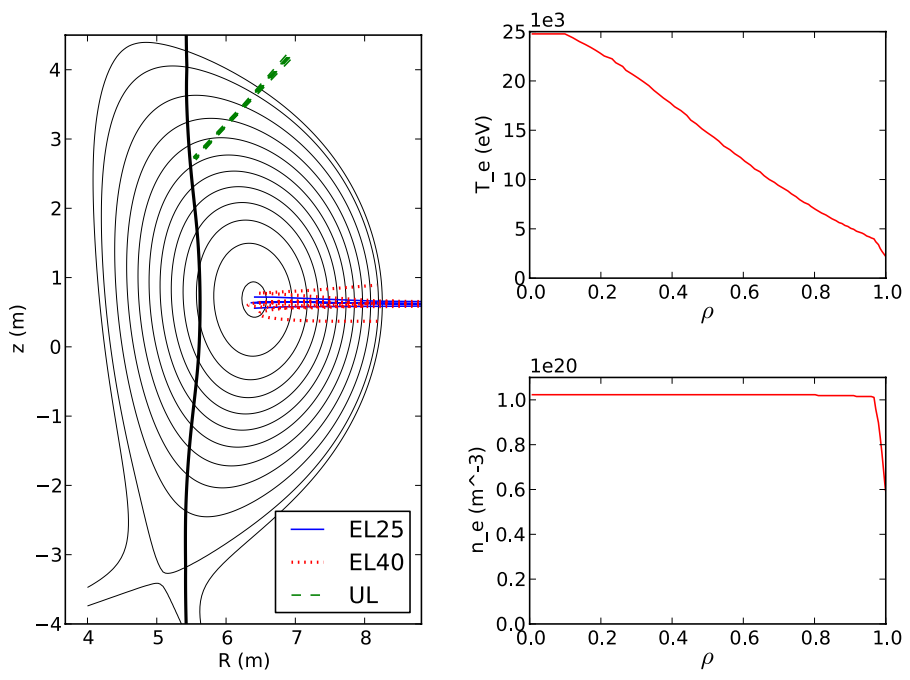

Fig. 2. Magnetic equilibrium (left), electron temperature profile (top right), and electron density profile (bottom right) of the ITER scenario used. The cold resonance at $170 \mathrm{GHz}$ is shown in the left plot (thick vertical line), with the beam-tracing computed by the GRAY code for the three launches considered.

Table 2. Launching conditions chosen for the benchmark.

\begin{tabular}{llcccccc}
\hline & Case & $R_{m}(\mathrm{~m})$ & $z_{m}(\mathrm{~m})$ & $\alpha\left(^{\circ}\right)$ & $\beta\left({ }^{\circ}\right)$ & $w_{0}(\mathrm{~m})$ & $d(\mathrm{~m})$ \\
\hline (i) & EL25 & 9.27 & 0.62 & 0 & 25 & 0.030 & 0.00 \\
(ii) & EL40 & 9.27 & 0.62 & 0 & 40 & 0.030 & 0.00 \\
(iii) & UL & 6.90 & 4.18 & 48 & 18 & 0.021 & 1.62 \\
\hline
\end{tabular}

from the UL of a beam focused in the plasma. The beam is aimed at the $q=3 / 2$ surface, and the focus is close to the resonance region. For all the cases, O-mode polarization is considered, with frequency $f=170 \mathrm{GHz}$, and input power $P_{0}=1 \mathrm{MW}$. The parameters for the three launching conditions tested are summarized in Table 2. Here and throughout all the paper, the poloidal and toroidal launching angles are defined as $\alpha=\tan ^{-1}\left(k_{0, z} / k_{0, R}\right)$ and $\beta=\sin ^{-1}\left(k_{0, \phi} / k_{0}\right)$, where $\left(k_{0, R}, k_{0, \phi}, k_{0, z}\right)$ are the wave vector components of the launched wave, in a cylindrical reference frame with the $z$ axis aligned with the tokamak symmetry axis.

\section{Results}

Before starting with the actual benchmarking, the zero-th step has been an extensive check of matching between ITM's and all codes sign conventions and definitions of physical quantities, to ensure that the input and output data were correctly interpreted and written by the codes. Although obvious, this step required quite a substantial effort, evidencing the fact that having a standardized data structure is not enough for a correct communication among different codes, its content must be handled properly too. After this initial checks, all the codes were run, reading the input data from the same CPOs dataset, and storing the results in a database, making use of the routines offered by the ITM framework. Since the main interest here was to test the possibility of minimizing the differences among the codes by taking advantage of the integrated modelling framework, then the same models for propagation, absorption, and current drive were used in all codes where possible. The cold dispersion relation was always used for propagation, while fully relativistic formulation was applied for absorption. For the codes in which 

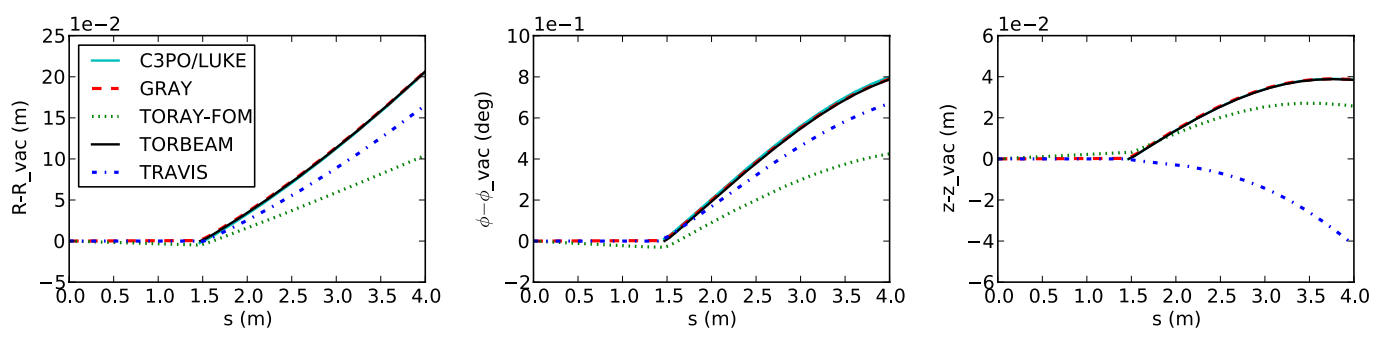

Fig. 3. Difference in $(R, \phi, z)$ coordinates (left, center, right) between the trajectories computed and the straight trajectory expected in vacuum, for the EL40 case. Vacuum-plasma transition is evident, about $1.5 \mathrm{~m}$ after the launching point.

Table 3. Summary of the results for current drive computation.

\begin{tabular}{lccccccccc}
\hline Code & \multicolumn{3}{c}{ EL25 } & \multicolumn{3}{c}{$\mathrm{EL} 40$} \\
$J\left(\mathrm{kA} / \mathrm{m}^{2}\right)$ & $\rho_{J}$ & $I(\mathrm{kA})$ & $J\left(\mathrm{kA} / \mathrm{m}^{2}\right)$ & $\rho_{J}$ & $I(\mathrm{kA})$ & $J\left(\mathrm{kA} / \mathrm{m}^{2}\right)$ & $\rho_{J}$ \\
\hline C3PO/LUKE & -41.1 & -109.3 & 0.18 & -19.8 & -5.91 & 0.54 & -6.74 & -17.8 & 0.81 \\
GRAY & -35.0 & -69.6 & 0.18 & -17.3 & -4.71 & 0.56 & -6.60 & -16.8 & 0.81 \\
TORAY-FOM & -29.0 & -63.4 & 0.18 & -16.4 & -4.61 & 0.56 & -5.21 & -13.0 & 0.81 \\
TORBEAM & -35.3 & -83.0 & 0.20 & -17.1 & -5.15 & 0.57 & -6.29 & -15.5 & 0.81 \\
TRAVIS & -33.3 & -66.0 & 0.18 & -16.3 & -4.58 & 0.56 & -6.20 & -14.2 & 0.80 \\
\hline
\end{tabular}

current drive calculation is based on the adjoint approach, the more accurate description including momentum conservation was selected. In the case of TORAY-FOM, the high speed limit model was used instead. In LUKE, the Fokker-Planck calculations were performed with a quasi-linear approach. Differences that cannot be avoided reside in the beam description, and in the handling of the vacuum to plasma transition: simple ray-tracing cannot describe diffraction effects, which are expected to play a role in the UL case where the beam is focused inside the plasma, and a different description of the plasma boundary may cause variations in the beam trajectory due to refraction.

Edge refraction has a large impact on beam propagation indeed. Figure 3 shows the difference among a straight trajectory and the trajectory of the beam center computed for the EL40 case: differences as large as $10 \mathrm{~cm}$ are observed among trajectories after a propagation length of $4 \mathrm{~m}$. The effect is amplified by the geometry of the case under test, which has high edge density gradient, and long path from boundary to absorption region (1.25-2.5 m, depending on the case). The high density value at the separatrix $n_{e \text { sep }} \sim 6 \times 10^{19} \mathrm{~m}^{-3}$ explains the smaller refraction shown by TORAY-FOM, which models a uniform scrape-off layer with density $n_{e \text {,sep }}$ up to the antenna. However, the influence of these discrepancies on computed power and current density profiles is still moderate, and the position of the profiles match within $\delta \rho<0.02$ in normalized radius units in all the cases, as can be seen in Table 3 and in Figure 4. Only in case of strongly focused beam, like in the UL case, the uncertainty may approach the profiles width. In general, good agreement is found in all the cases, with differences in total current $\left|\delta I_{c d} / I_{c d}\right|<15 \%$, and with peak values of power density $d P / d V$ and driven current density $J$ typically matching within $10 \%$. In the EL25 case some interaction is seen at $\rho \sim 0.1$ only with TORAY-FOM. The actual cause is still under investigation, a possible explanation being that absorption is shifted inwards due to smaller refraction at the edge. C3PO/LUKE and TORBEAM show profiles more peaked by $10-15 \%$, possibly due to quasi-linear effects (LUKE), or to the beam model used (TORBEAM). In the EL40 case interaction occurs at mid radius, doppler broadening dominates the effect of finite beam size in the determination of the profiles width, and all the codes here agree very well. Finally, in the UL case, despite the focused beam, the profiles are reasonably well reconstructed also by ray-tracing codes, giving results comparable to those obtained by the codes which account for diffraction effects. The very narrow profiles make the displacement among the profiles, that is comparable to that found 

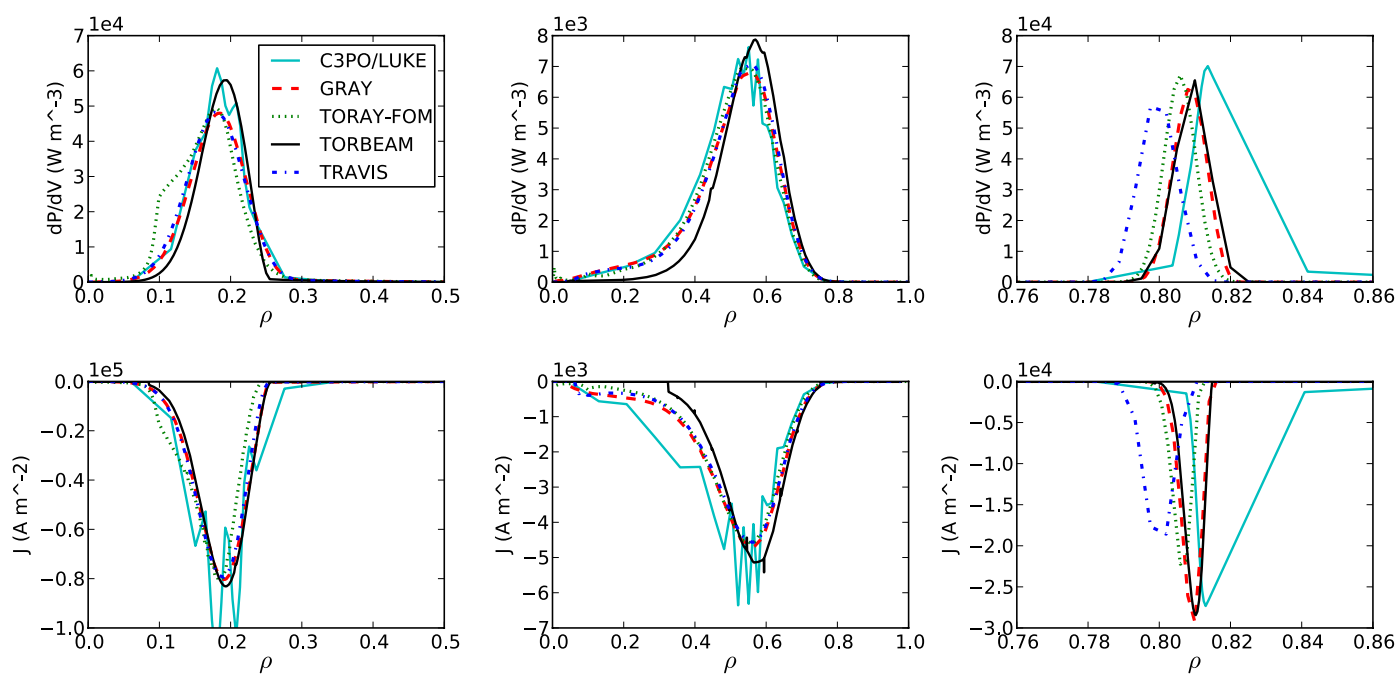

Fig. 4. Power density (top) and driven current density (bottom) computed for the launching conditions of Table 2: EL25 (left), EL40 (center) and UL (right).

in the other cases, more apparent. In some cases, the exact profiles reconstruction is limited by the coarseness of the radial grid, while the choice of a too fine radial grid is the cause of the oscillations in the profiles given by $\mathrm{C} 3 \mathrm{PO} / \mathrm{LUKE}$ for the EL cases.

\section{Conclusions}

ITM-TF framework proved to be a valuable environment to benchmark codes sharing a similar physics. The benchmarking work put in evidence the necessity, for any integrated modelling framework, to have a clear and unambiguous definition of all the physical quantities on top of a standardized interface. All the European ECH/ECCD codes ported to the framework demonstrated to handle reasonably well even the more demanding test cases, like central ECCD at high temperature, and beam focused close to the resonance region.

This work was carried out within the framework of the European Fusion Development Agreement Task Force on Integrated Tokamak Modelling, under the IMP5 project "Heating, Current Drive and Fast Particles". The views and opinions expressed herein do not necessarily reflect those of the European Commission.

\section{References}

1. F. Wagner, et al, Plasma Phys. Control. Fusion 52 (2010) 124044

2. F. Imbeaux, et al, Comp. Phys. Commun. 181 (2010) 987

3. http://kepler-project.org

4. D. Farina, Fusion Sci. Technol. 52 (2007) 154

5. E. Westerhof, Implementation of TORAY at JET (Rijnhuizen Report, 1989) RR-89-183

6. E. Poli, et al, Comp. Phys. Commun. 136 (2001) 90

7. R. Prater, et al, Nucl. Fusion 48 (2008) 035006

8. N. B. Marushchenko, et al, J. Plasma Fusion Res. 2 (2007) S1000

9. Y. Peysson, et al, Plasma Phys. Control. Fusion 54 (2012) 045003

10. N. B. Marushchenko, et al, Fusion Sci. Technol. 55 (2009) 180

11. Y. R. Lin-Liu, et al, Phys. Plasmas 10 (2003) 4064 